\title{
THE EFFECT OF EMOTIONAL INTELLIGENCE ON THE DiVERSITY CLIMATE AND INNOVATION
} CAPABILITIES

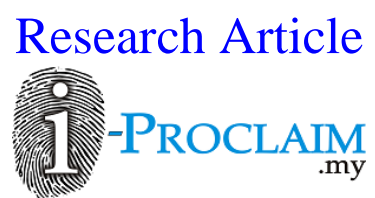

Asia Pac. j. energy environ.

\section{Harish Paruchuri ${ }^{1^{*}}$, ABM Asadullah ${ }^{2}$}

\author{
${ }^{1}$ Senior AI Engineer, Department of Information Technology, Anthem, Inc., USA \\ ${ }^{2}$ Department of Economics and Management Sciences, International Islamic University Malaysia, Kuala Lumpur, MALAYSIA
}

*Email for Correspondence: harish.paruchuri@ anthem.com

Abstract

This study explores the relationship between emotional intelligence, a diverse work environment, a creative climate, and an innovative corporate culture and capabilities. To get a large enough sample, the questionnaire was used. Based on our findings, it appears that emotional intelligence has an impact on a diverse workplace climate. A diverse workforce and an innovative service mix are inextricably linked. Based on our findings, it appears that diversity in an organization's innovation culture is directly related to the pace of innovation in that organization. Our goal is to provide additional insight into existing research in the public sector. A company's emotional intelligence practices are implemented, and the employees' emotional intelligence skills are taught. The study also encourages the implementation of policies to help deal with climate change and innovation diversity. These policies include diverse input in innovation decision-making. This advice can be used by everyone, helping organizations attain better technical results. Service innovation capabilities are influenced by factors such as emotional intelligence, a diverse work environment, and an innovative business culture.

Key words

Diversity climate, invention abilities, emotional intelligence, technical outcomes

\section{INTRODUCTION}

Innovation in the public sector has a different set of conditions than that of private enterprise. One of those conditions is the atmosphere that fosters and empowers innovation as a means of tapping into the social and economic value contained in knowledge and the necessity of creative thinking for inventing improved and new services in the public sector. It promotes creativity and innovation by eradicating activities that may hinder employees in the public sector from facilitating service innovation capabilities. Innovative leadership and employee cultures both encourage leaders and employees to seek out and implement innovative services to the public (Movva et al., 2012). This wide range of services makes it possible for the public sector to accommodate creativity and diverse business ideas from all industries, and this leads to new service innovations.

The antecedents of conduct, such as attributions, perceptions, and attitudes, are influenced by emotions. Emotions are people's everyday emotional experience, such as feelings of excitement, and that impacts behavior, as well as the people who interact with that individual. Empathy and managing one's emotions are two important emotional intelligence skills that people should have to handle daily stress, get along with others, solve conflicts, overcome the challenge, and make effective communications. Employers and employees benefit from practicing emotional intelligence because of their culture and diversity. It is because emotional intelligence recognizes the significance of those emotions. Each of these four essential elements has a huge impact on public sector innovation: motivation, selfawareness, empathy, social skills, and self-regulation (Vadlamudi, 2016). Demand for service has risen while the public sector continues to pursue service innovation. A country's economic growth is highly dependent on the service sector. Private sector service innovation capabilities are emerging as a topic to be conquered.

Diversity in the public sector stems from emotional intelligence as individuals can manage their emotions and utilize those emotions to accommodate various people's views and new ideas. Additionally, emotional intelligence enables 
public sector organizations to generate innovative service capability innovations while fostering employees' personal and professional growth. Accordingly, emotional intelligence also supports designing a public sector workplace that allows for the implementation of new service innovation capabilities. Recent studies show that those who are seen as being very emotionally intelligent have a high likelihood of success and outstanding performance. Stakeholders external to the organization must have high levels of emotional intelligence (Paruchuri, 2017).

As the research on emotional intelligence involves analyzing samples from diverse cultures, this data is collected to allow for technical accumulation. This research seeks to fill the gap in the existing literature, as the public sector in the USA is increasingly required to deliver greater levels of service quality. They seek to implement ways to increase the ability to innovate with services. When considering people's emotions, emotion is necessary in the workplace, allowing for better critical thinking and problem-solving. Employees in the public sector require the ability to understand and utilize their emotions when dealing with a wide range of views, as they are constantly exposed to a diverse range of points of view (Vadlamudi, 2015). We also consider it to be a plus if a leader has an effective method for accessing their team's emotions, since it allows them to perceive and process their team's emotions and use that information to make decisions and communicate those decisions and emotions to their team members. This research will provide guidelines to practitioners who wish to improve service innovation capability in public organizations in the USA by understanding the role of emotional intelligence, diversity climate, and innovation culture.

\section{RESEARCH GaP}

Only in the last two decades has increasing attention been given to diversity issues. The evidence presented shows that the last decade has seen the most research on perceived diversity climate and only a handful of the studies utilized diversity climate as a variable (Paruchuri, 2015). In relation to team performance, job satisfaction, and organizational commitment, there have been studies which indicate that employees' perceptions of diversity affect company productivity and success. There are few or no studies dealing with the personal or individual factors that contribute to perceptions of climate diversity worldwide. Consequently, this research gap is needed to carry out the study's true objective, which is to discover the correlation between emotional intelligence and workplace diversity.

\section{The Mediating Role of Diversity Climate}

Service innovation capabilities can be gained by the recognition of services as key non-technological influences on product creation, including customer integration. So, in order to maximize service innovation capabilities, the organization can rely on diversity climate for higher quality services and customer relationships. An effective way to maximize service innovation capabilities is through knowledge sharing, networking, and excellent team organization. Knowledge sharing, networking, and organization are all advantages of emotional intelligence when used in the public sector (Ganapathy \& Neogy, 2017). There is a connection between diversity in climate and service innovation, as it facilitates employees' knowledge and skills, allowing them to originate new and valuable ideas.

Emotional intelligence, which Bar-On (2006) has defined as a collection of various emotional and social competencies, skills, and facilitators, assists in performance in a variety of ways. People of all walks of life interact with one another as customers in the public sector, and these areas are vital to the public sector in this regard. They are expected to serve everyone they can until they reach their fullest potential. Additionally, people who have developed an emotional intelligence skill set are better able to cope with daily pressures, both at work and in their personal lives. Mentally intelligent people are better able to deal with everyday stress, thus enabling them to find better ways to increase efficiency in the workplace, which culminates in innovative service capabilities.

\section{The Moderating Role of InNovation Culture}

An organization's tendency to innovate is related to its innovation culture Creating an innovative work environment in the public sector that encourages creative thinking will lead to improved services for the industry. Empathy, motivation, self-awareness, and social skills impact innovation practices in the public sector. Emotional intelligence is necessary to serve diverse customers in the public sector (Ganapathy, 2017). Public sector workers are also diverse. As a result, emotional intelligence is required to facilitate collaboration and generate innovative service capabilities by fostering diverse opinions. Encouraging employees to speak up and take risks naturally aids in the creation of an innovation culture.

The public sector often finds itself faced with problems that hinder it from competing and offering quality services to rival private organizations. Investing in service innovation capabilities can improve services and increase customer retention. A business's service innovation capabilities are impacted by several factors which affect employee capacity and willingness to innovate. The attitude of emotional intelligence is conducive to creating an optimistic work environment and enhancing productivity. 
An organization's leadership can impact the capabilities needed for innovation and creativity. Emotional intelligence impacts leadership styles and corporate management teams, allowing them to feel their team's emotions and empathize with them. Leadership is equally important in business because leaders affect employee job satisfaction by encouraging their employees and providing a relaxed and open atmosphere. Flexible work environments enable employees to pursue new ideas because there are no ramifications. In order to develop service innovation capabilities in the public sector, employees should be provided with an atmosphere that fosters collaboration and encourages the freedom to explore new methods. Successful leaders not only understand their employees' needs and emotions, but they are also models of how employees are expected to behave and perform. Connecting with people's emotions allows leaders to use motivational techniques that increase employee morale and engagement, resulting in greater interdepartmental collaboration and innovation. A company's leadership influences both the company's organizational culture and innovation capabilities (Ganapathy, 2016). Employees develop social competencies to adapt to various situations and customer requests because of their emotional intelligence.

An innovation culture encourages employees to be creative and to freely share their innovative ideas, which helps for continuous improvement. Transformational leadership empowers leaders to establish meaningful relationships with their employees by offering essential support. Leaders' willingness to support their employees has the side effect of increasing employee loyalty and encouraging innovation. So, therefore, to become transformational leaders who influence and inspire their employees, employees must first cultivate their emotional intelligence. The ability to evaluate problems analytically and develop a proper action plan also correlates with emotional intelligence. Quick and analytical decision-making is a necessity in providing excellent customer service.

Leadership and organizational culture determine employee empowerment, as suggested by the authors. When leaders empower employees to use their knowledge and skills to develop and implement solutions, they enable service innovation capabilities. Delegating tasks and offering workers some decision-making authority help emotionally intelligent leaders empower their employees. An understanding of one's capabilities coupled with a tailored approach to tasks based on those abilities is critical for effective human resource management. Employees who are emotionally intelligent are less likely to be affected by conflicts and task uncertainty at work, including passive-aggressive behavior.

\section{METHOD}

The cross-sectional data were acquired from public sector service organizations in the United States, as the study focuses on the public sector. As a result, the sample is made up of people who work in the public sector. The data was obtained from individuals in several functional areas across multiple public sector organizations in order to obtain enough heterogeneity to ensure the construct relationships' stability. The United States Government website included a list of public service sector organizations. Five organizations were chosen at random from the list to be approached, and only one agreed to participate in the survey. Employees who had been with the company for at least two years and worked at a different level to assess service innovation capabilities were included in the study. All things were rated on a 5-point Likert scale, with 1 being the lowest and 5 being the highest (strongly disagree to strongly agree). The variables were measured using the scales listed below.

\section{CONFIRMATORY FACTOR ANALYSIS}

AMOS was evaluated for various indexes to see how well the model fit. First, we will look at the chi-square index (CMIN). If the chi-square is insignificant, the model is accepted. The observed covariance matrix resembles the predicted covariance matrix (the matrix predicted by the model). Several writers have proposed using this measure of fit. Except for Uls and Sls, the ratio should approximate the model.

In addition, the study examined the Goodness of Fit index (accounting for the total variance) and the RSMEA index. The best fit has an RMSEA of less than 0.08. Until the early 1990s, an RMSEA of 0.05 to 0.10 was considered good fit, and values above 0.10 indicated poor fit (Movva et al., 2012). The CFI examines model fit by comparing the data with the model, correcting for sample size biases built into the chi-squared test of model fit and the normed fit index. A greater fit has a CFI value greater than zero.

\section{NSW Public Sector Context}

A review of executive competency in the NSW public sector found four areas where work needs to be done, including:

1. High-level ICT management;

2. Strategic financial management;

3. High-level contract management; and 


\section{Strategic human resource leadership.}

It's clear that the technological and cognitive aspects are being prioritized. According to Newman et al. (2009), it is more necessary for public officials to work in a humane and caring manner than to be more efficient. As previously said, this sector has been regarded as having inadequate performance management and an inability to have unpleasant dialogues with employees. Managers' avoidance of these events and dialogues suggests a lack of emotional competency.

\section{NSW Public Sector Capability Framework}

There are five different public sector capability groups and 20 core capabilities within that list. A capability is adaptable to different situational contexts. Fundamental, intermediate, adept, advanced, and advanced are the levels. Each behavioral indicator set represents the degree of knowledge, skills, and abilities required to be successful. There is a 34-page document posted on the PSC website that goes into detail on the five groups and relevant competencies with examples of their respective behaviors.

1. Personal attributes:

- Display resilience and courage: be open and honest, prepared to express your views, and willing to accept and commit to change;

- Act with integrity: be ethical and professional, and adhere to the public sector values;

- Manage self: show drive and motivation, a measured approach and a commitment to learning; and

- Value diversity: show respect for diverse backgrounds, experiences and perspectives.

2. Relationships:

- Communicate effectively: communicate clearly, actively listen to others and respond with respect;

- Commit to customer service: provide customer centric services in line with public service and organizational objectives;

- Work collaboratively: collaborate with others and value their contribution; and

- Influence and negotiate: gain consensus and commitment from others and resolve issues and conflicts.

3. Results:

- Deliver results: achieve results through efficient use of resources and a commitment to quality outcomes;

- Plan and priorities: plan to achieve priority outcomes and respond flexibly to changing circumstances;

- Think and solve problems: think, analyses and consider the broader context to develop practical solutions; and

- Demonstrate accountability: be responsible for own actions, adhere to legislation and policy and be proactive to address risk.

4. Business enablers:

- Finance: understand and apply financial processes to achieve value for money and minimize financial risk;

- Technology: understand and use available technologies to maximize efficiencies and effectiveness;

- Procurement and contract management: understand and apply procurement processes to ensure effective purchasing and contract performance; and

- Project management: understand and apply effective planning, coordination and control methods.

5. People management - only applicable to those managing staff:

- Manage and develop people: engage and motivate staff and develop capability and potential in others;

- Inspire direction and purpose: communicate goals, priorities and vision and recognize achievements;

- Optimize business outcomes: manage resources effectively and apply sound workforce planning principles; and

- Manage reform and change: support, promote and champion change, and assist others to engage with change.

Source: NSW Public Service Commission (2013).

\section{RESULTS AND DISCUSSION}

Only six of the 490 behavioral indicators listed in the framework mention emotional elements in three of the 20 competencies. An emotional capacity isn't a critical backbone capability that underpins the competencies presented here. The review focuses on technical and managerial topics, emphasizing "what" rather than "how". Public sector management remains linked to traditional ways of thinking. Ujwala et al. (2012) noted that leaders can have the "what" but lack the "how" skills. How skills are significant in interpersonal interactions. 
Additionally, people management is focused on the role of direct reports because these competencies are not important in daily interactions and in project teams where no formal authority exists. A coordinated, cooperative effort is required for the success of an organization (Vadlamudi, 2018). As informal leadership impacts interpersonal relationships and influence, organizations may be able to leverage that asset to improve morale and productivity (Miner, 2013). This strongly suggests that employees' work-related competence abilities can benefit everyone. They combine complementary competencies, further improving the organization's overall performance.

Empathy, motivation, and relationship management are fundamental characteristics of these areas. Emotions are core to human interactions, which impact employee behavior and productivity (Neogy \& Paruchuri, 2014). Influence occurs in a variety of relationships, both formally and informally. A prime capability, "Manage and Develop People," focuses on job performance, performance development, and performance frameworks.

Acknowledged already, change results from new assumptions. Capability frameworks are artifacts that reinforce desired assumptions. The NSW sector's capability framework is a secondary reinforcement mechanism that reinforces the underlying assumptions that drive attitudes and behaviors. Embedding mechanisms include competencies that are systemically measured and controlled. Thus, the technical and managerial components will be emphasized further (Narayana et al. 2012). Emotional capability is consistently observed to have the capacity to engage and obtain a commitment from employees. This will facilitate the realization of cultural change and enhance the relevant competencies required for this continually evolving sector.

\section{Limitation AND FUTURE DiRECTIONS}

This study has some limits. Social desirability bias impacted the data that was used for analysis. They are also limited to the public sector, which restricts their generalizability and validity. Secondly, the study scope is limited to the United States, which limits the findings' generalizability (Vadlamudi, 2017). Thirdly, the results are based on cross-sectional data without taking into consideration any interventions or evaluations of their effects on service innovation capabilities. These practices support emotional intelligence, diversity, and innovation in public organizations.

Future researchers can avoid these limitations by using different methodologies or by repeating the same methodology in different locations. The same methodology may be applied to other sectors in other countries and other regions. For example, the researchers can use longitudinal data in the USA's public sector to confirm the findings of the present study and to acknowledge how emotional intelligence impacts the diversity climate and the role it plays in moderating the outcomes of diversity climate and service innovation culture. Future researchers should conduct similar studies in the private sector and SMEs, as well as qualitative studies to gather data.

\section{CONCLUSION}

It is intended that this research study will be of assistance to scholars, students, academicians, and management practitioners. In today's highly competitive business environment, organizations must go above and beyond to not only attract a diverse workforce but also to maximize their potential and motivate them to achieve corporate goals. Organizations should be aware of the impact of each employee's emotional intelligence on his or her work behavior and performance. Priority should be given to making the organization's diversity policy and programs transparent and inclusive of all groups. HR policies should be reinforced by attracting the right workforce (diversity) and offering enough sensitivity training so that they can harness and function as a cohesive unit. Because a varied staff helps the company flourish, it also attracts and benefits customers, society, and the country as a whole.

Hiring people with high emotional intelligence and providing training to present employees to improve their emotional intelligence are two of the research's key practical contributions. Furthermore, the study recommends strategies to promote a varied climate and innovation culture in public organizations, as well as diverse input in innovation decision-making, which will result in improved service innovation capabilities. These recommendations can be implemented by public organizations, which should help them increase their service innovation skills and hence improve organizational outcomes.

\section{REFERENCES}

Bar-On, R. (2006). The Bar-On model of emotional-social intelligence. In P. Fernández-Berrocal and N. Extremera (Guest Editors), Special Issue on Emotional Intelligence. Psicothema, 17.

Ganapathy, A. (2015). AI Fitness Checks, Maintenance and Monitoring on Systems Managing Content \& Data: A Study on CMS World. Malaysian Journal of Medical and Biological Research,2(2), 113-118. https://doi.org/10.18034/mjmbr.v2i2.553 
Ganapathy, A. (2016). Speech Emotion Recognition Using Deep Learning Techniques. ABC Journal of Advanced Research, 5(2), 113-122. https://doi.org/10.18034/abcjar.v5i2.550

Ganapathy, A. (2017). Friendly URLs in the CMS and Power of Global Ranking with Crawlers with Added Security. Engineering International, 5(2), 87-96. https://doi.org/10.18034/ei.v5i2.541

Ganapathy, A., \& Neogy, T. K. (2017). Artificial Intelligence Price Emulator: A Study on Cryptocurrency. Global Disclosure of Economics and Business, 6(2), 115-122. https://doi.org/10.18034/gdeb.v6i2.558

Movva, L., Kurra, C., Koteswara Rao, G., Battula, R. B., Sridhar, M., \& Harish, P. (2012). Underwater Acoustic Sensor Networks: A Survey on MAC and Routing Protocols. International Journal of Computer Technology and Applications, 3(3).

Narayana, S. L., Suneetha Devi J., Bhargav Reddy I., Harish Paruchuri. (2012). Optimizing Voice Recognition using Various Techniques. CiiT International Journal of Digital Signal Processing, 4(4), 135-141

Neogy, T. K., \& Paruchuri, H. (2014). Machine Learning as a New Search Engine Interface: An Overview. Engineering International, 2(2), 103-112. https://doi.org/10.18034/ei.v2i2.539

Newman, M.A., Guy, M.E. and Mastracci, S.H., (2009). Beyond cognition: affective leadership and emotional labor. Public Administration Review, 69(1), 6-20.

NSW Public Service Commission, (2013). Capability framework. NSW Public Service Commission, Sydney, available at: www.psc.nsw.gov.au/Sector-Support/Capability-Framework, (6 September 2014).

Paruchuri, H. (2015). Application of Artificial Neural Network to ANPR: An Overview. ABC Journal of Advanced Research, 4(2), 143-152. https://doi.org/10.18034/abcjar.v4i2.549

Paruchuri, H. (2017). Credit Card Fraud Detection using Machine Learning: A Systematic Literature Review. $A B C$ Journal of Advanced Research, 6(2), 113-120. https://doi.org/10.18034/abcjar.v6i2.547

Ujwala, D., Ram Kiran, D. S., Jyothi, B., Fathima, S. S., Paruchuri, H., Koushik, Y. M. S. R. (2012). A Parametric Study on Impedance Matching of A CPW Fed T-shaped UWB Antenna. International Journal of Soft Computing and Engineering, 2(2), 433-436.

Vadlamudi, S. (2015). Enabling Trustworthiness in Artificial Intelligence - A Detailed Discussion. Engineering International, 3(2), 105-114. https://doi.org/10.18034/ei.v3i2.519

Vadlamudi, S. (2016). What Impact does Internet of Things have on Project Management in Project based Firms?. Asian Business Review, 6(3), 179-186. https://doi.org/10.18034/abr.v6i3.520

Vadlamudi, S. (2017). Stock Market Prediction using Machine Learning: A Systematic Literature Review. American Journal of Trade and Policy, 4(3), 123-128. https://doi.org/10.18034/ajtp.v4i3.521

Vadlamudi, S. (2018). Agri-Food System and Artificial Intelligence: Reconsidering Imperishability. Asian Journal of Applied Science and Engineering, 7(1), 33-42. Retrieved from https://journals.abc.us.org/index.php/ajase/article/view/1192 